\title{
Endoscopic Ultrasound-Guided Liver Biopsy in the Hands of a Chest Physician
}

\author{
Markus Fally ${ }^{\mathrm{a}}$ Rafi Nessar ${ }^{\mathrm{a}}$ Nille Behrendt ${ }^{\mathrm{b}}$ Paul Frost Clementsen ${ }^{\mathrm{c}, \mathrm{d}}$ \\ Departments of ${ }^{\mathrm{a}}$ Internal Medicine and ${ }^{\mathrm{b}}$ Pathology, Zealand University Hospital Roskilde, Roskilde, \\ 'Department of Respiratory Medicine, Gentofte University Hospital, Hellerup, and ${ }^{\mathrm{d}}$ Copenhagen Academy for \\ Medical Education and Simulation (CAMES), Rigshospitalet, Copenhagen, Denmark
}

\section{Established Facts}

- Endoscopic ultrasound-guided fine needle aspiration with the gastrointestinal endoscope from lesions below the diaphragm is well described.

- Liver biopsy using transesophageal bronchoscopic ultrasound-guided fine needle aspiration (EUS-BFNA) has never been described before and biopsies of infradiaphragmatic lesions for staging lung cancer using this technique are not considered to be standard.

\section{Novel Insights}

- We have demonstrated that EUS-B-FNA from a liver metastasis in a patient with lung cancer is possible and can be of high diagnostic value.

\section{Key Words}

Bronchoscopy · Endobronchial biopsy · Endobronchial ultrasound - Endoscopic ultrasound - Endoscopic ultrasound-guided fine needle aspiration · Lung cancer . Lung cancer staging

\footnotetext{
Abstract

Liver biopsy using transesophageal bronchoscopic ultrasound-guided fine needle aspiration (EUS-B-FNA) has never been described before and biopsies of infradiaphragmatic lesions using this technique are not considered to be standard. A patient suspected for primary lung cancer with mul-
}

tiple lesions in the liver was referred to our department. We conducted bronchoscopy and endobronchial ultrasoundguided (EBUS) biopsy from several enlarged mediastinal lymph nodes. Thereafter, we conducted EUS-B-FNA from a lesion in the left liver lobe. Pathology showed that the liver lesion represented a metastasis from a pulmonary adenocarcinoma. Bronchoscopy and EBUS samples were not able to establish diagnosis. We hereby demonstrated that a diagnostic EUS-B-FNA from a liver metastasis in a patient with lung cancer is possible. This underlines that chest physicians should not forget the esophagus when staging lung cancer.

(c) 2016 S. Karger AG, Basel

Markus Fally, MD

Department of Internal Medicine, Zealand University Hospital Roskilde Sygehusvej 10

DK-4000 Roskilde (Denmark)

E-Mail fally@ dadlnet.dk 


\section{Introduction}

Endoscopic ultrasound-guided fine needle aspiration (EUS-FNA) with the gastrointestinal (GI) endoscope from the left adrenal gland for staging of lung cancer patients is well described as an accurate method $[1,2]$. In recent years, chest physicians have started using an endoscope designed for endobronchial ultrasound (EBUS) in the esophagus (EUS-B) for detection and biopsy of lesions both above and below the diaphragm including the left adrenal gland [3-5]. However, EUS-BFNA from a liver metastasis has never been described before.

\section{Case Report}

A 73-year-old female was referred with a tumor in the lower lobe of the right lung, multiple lesions in the liver, enlarged mediastinal lymph nodes, and an enlarged left adrenal gland. The most important computed tomography (CT) findings are presented in figures 1 and 2. Ultrasound-guided percutaneous liver biopsy could not be performed due to the lesions' location, size and movement during respiration. Bronchoscopy with brush biopsy and bronchial lavage was performed followed by EBUSguided transbronchial needle aspiration (TBNA) from several lymph node stations in the mediastinum. In the same session we inserted the EBUS endoscope (Olympus BF-UC180F linear ultrasound bronchoscope with a curved linear assay, 80 degrees view and a working length of $600 \mathrm{~mm}$ ) in the esophagus. A lesion in the left liver lobe was easily identified approximately $45 \mathrm{~cm}$ from the upper row of teeth by turning the transducer counterclock-

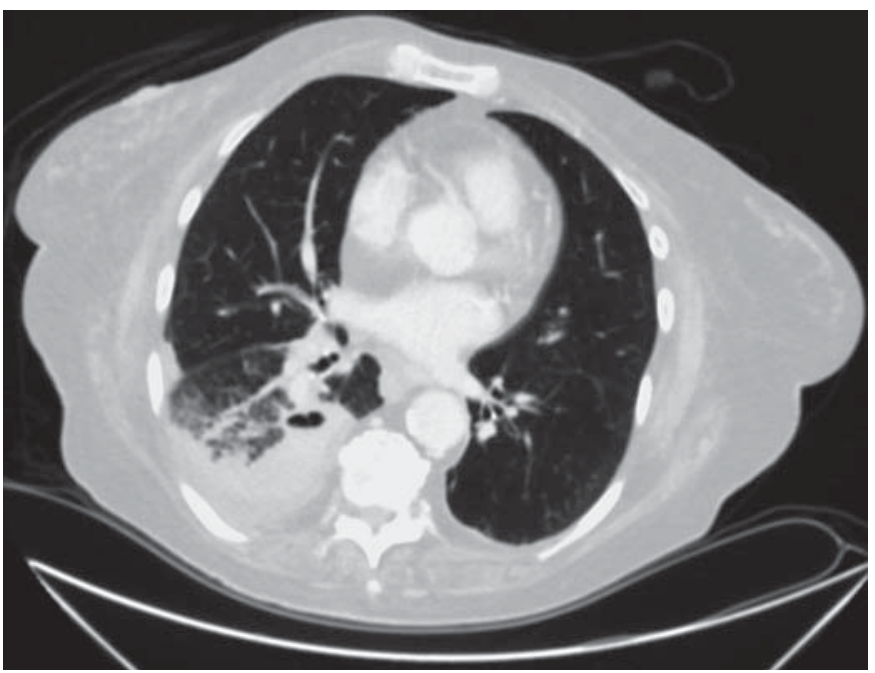

Fig. 1. Suspected primary tumor in the lower lobe of the right lung. wise. It presented approximately $2 \mathrm{~cm}$ in size, with an indistinct margin and heterogeneous echogenicity. No blood vessels were seen in or close to the lesion. An FNA was performed with a 22-gauge needle.

Cytology and immunohistochemistry were diagnostic for a liver metastasis from a primary pulmonary adenocarcinoma (non-small-cell lung carcinoma). Cells representing liver tissue were also seen in the aspirate (fig. 3). The cells expressed thyroid transcription factor-1 (TTF-1) and cytokeratin 7 (CK7) and lacked CK20, as well as transcription factors p63 and p40. EUS-BFNA from the left adrenal gland showed similar malignant cells, whereas bronchoscopy and EBUS-TBNA did not reveal malignancy. The conclusion was that the patient was suffering from stage IV lung cancer.

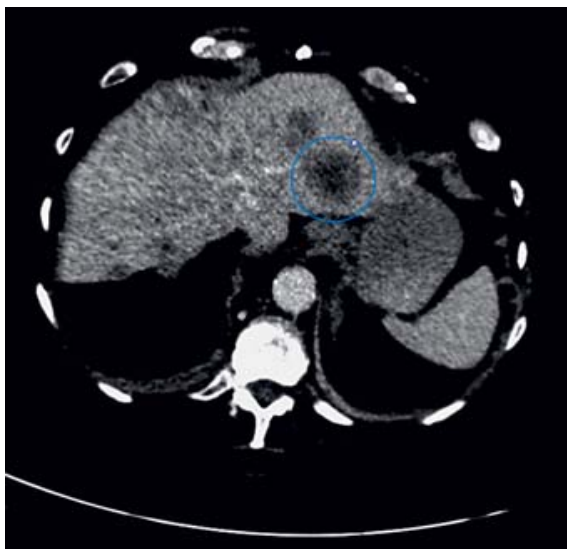

Fig. 2. Metastatic lesion in the left liver lobe - axial.

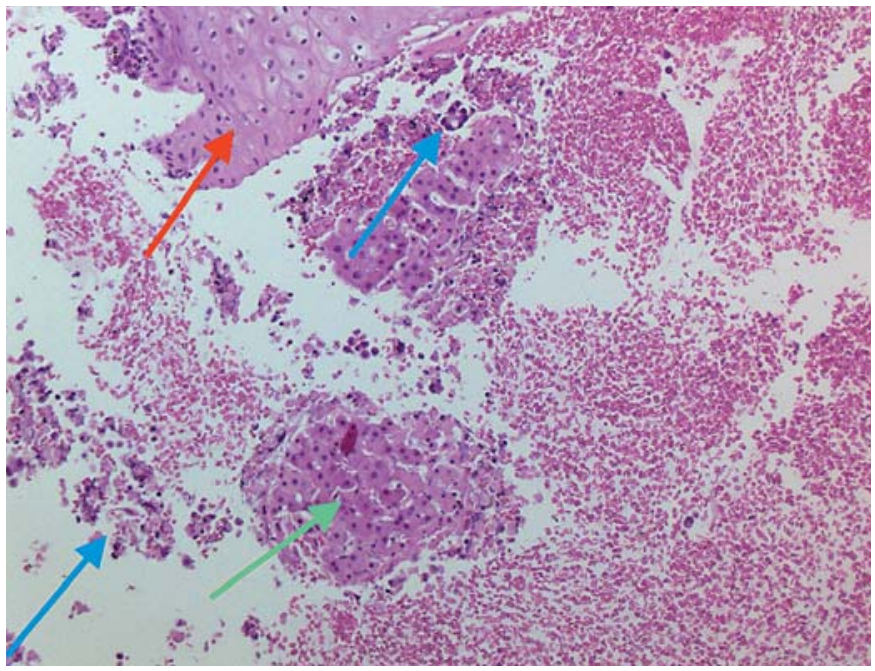

Fig. 3. Liver biopsy showing cells of a metastatic pulmonary adenocarcinoma (blue), normal esophageal squamous epithelia (red) and hepatocytes (green). Hematoxylin and eosin stain. $\times 10$. 


\section{Discussion}

Accurate staging of lung cancer is mandatory for planning of optimal treatment. Surgery or radiotherapy with curative intent is advised in the case of localized disease. Imaging by $\mathrm{CT}$ and/or positron emission tomography should be obtained to characterize the primary lung lesion, the mediastinum, and to search for metastases, but additional tissue staging is frequently required to confirm or exclude metastatic involvement, for example if a lesion in the liver is suspected of malignancy [1-6].

There are several ways of performing liver biopsy. Percutaneous liver biopsy can be done image guided using ultrasonography or CT (16-, 18- or 20-gauge needles). Furthermore, a transjugular approach via the superior caval and the hepatic vein has been described [7].

Endoscopic transesophageal ultrasound using the GI scope (EUS) has emerged as an alternative to obtain liver biopsy with a low risk of adverse events and the results and complications are well described. The procedure gives access to real-time visualization. Either a 19-gauge spring-loaded device to obtain a histological sample or a
22 -gauge needle for FNA can be used. The left liver lobe is accessible from the stomach and the right lobe from the duodenum [7].

For lung cancer staging, EUS-FNA is useful in combination with EBUS-TBNA. In short, EUS is excellent for the left and lower paraesophageal structures plus structures under the diaphragm, while EBUS provides access to structures close to the large airways on both sides [5].

Chest physicians have traditionally been reluctant to perform transesophageal endoscopic ultrasound using the big GI scope [6]. A way to overcome this psychological barrier is to let the chest physician perform EUS with the smaller EBUS endoscope, the so called EUS-B procedure, even if the equipment is not designed for the purpose and therefore probably inferior compared to EUSFNA with the conventional GI scope.

The present case report shows that EUS-B-FNA is possible not only from the left adrenal gland, but also from a metastasis in the liver. In conclusion, we recommend that chest physicians performing bronchoscopy and EBUSTBNA also consider performing EUS-B-FNA from lesions below the diaphragm including the left liver lobe.

\section{References}

>1 Schuurbiers OC, Tourgny KG, Schoppers HJ, Dijkman BG, Timmers HJ, de Geus-Oei LF, Grefte JM, Rabe KF, Dekhuijzen PN, van der Heijden HF, Annema JT: EUS-FNA for the detection of left adrenal metastasis in patients with lung cancer. Lung Cancer 2011;73:310315.

$>2$ Bodtger U, Vilmann P, Clementsen P, Galvis E, Bach K, Guldhammer Skov B: Clinical impact of endoscopic ultrasound-fine needle aspiration of the left adrenal masses in established or suspected lung cancer. J Thorac Oncol 2009;4:1485-1489.
-3 Annema JT, et al: EUS-B-FNA versus EUSFNA for left adrenal gland analysis in lung cancer patients. Ongoing study, ClinicalTrials.gov.

$\checkmark 4$ Crombag L, Bonta P, Annema J: Left adrenal gland analysis using the EBUS scope: a feasibility study in lung cancer patients. Eur Respir J 2015;46(suppl 59):PA779.

5 Vilmann P, Clementsen PF, Colella S, Dumonceau J-M, Herth FJ, Larghi A, Siemsen M, Vasquez-Sequeiros E, Hassan C, Crombag L, Konge L, Annema J: Combined endobronchial and esophageal endosonography for the di- agnosis and staging of lung cancer: a clinical guideline by the European Society of Gastrointestinal Endoscopy (ESGE), European Respiratory Society (ERS) and European Society of Thoracic Surgeons (ESTS). Endoscopy 2015;47:545-559.

6 Vilmann P, Clementsen PF: Combined EUS and EBUS are complementary methods in lung cancer staging. Do not forget the esophagus. Endosc Int Open 2015;3:E300-E301.

$>7$ Parekh PJ, Majithia R, Diehl DL, Baron TH Endocopic ultrasound-guided liver biopsy. Endosc Ultrasound 2015;2:85-91. 